Series A

I. MATHEMATICA

543

\begin{abstract}
BEMERKUNG ÜBER
DIE SINGULARITÄT VON LÖSUNGEN

DER ERSTEN RANDWERTAUFGABE

HYPERBOLISCHER DIFFERENTIALGLEICHUNGEN
\end{abstract}

BY

JOACHIM JAENICKE

H E L S N K I 1973

S U O M A L A I N E N TIEDEAKATEM I A

https://doi.org/10.5186/aasfm.1973.543 
Copyright (c) 1973 by Academia Scientiarum Fennica ISBN $951-41-0111-1$

Vorgelegt am 12. Februar 1973 


\section{Bemerkung über die Singularität von Lösungen der ersten Randwertaufgabe hyperbolischer Differentialgleichungen}

Für die erste Randwertaufgabe der Gleichung $u_{x y}=0$ über geeignete Zweieckgebiete gibt G. Fichera [1] Aussagen über das Verhalten einer Lösung in einer Ecke an. Hier wird gezeigt, wie diese Aussagen speziell auf die Funktionalgleichung der periodischen Funktionen und einer expliziten Darstellung des additiven singulären Anteils einer Lösung führen.

Sei $G$ ein Gebiet der $(x, y)$-Ebene, das zwischen $x=0$ und $x=b>0$ liegt mit der $x$-Achse als untere Berandung und einer Kurve $y=h(x)$ als obere Berandung mit

$$
\begin{gathered}
h \in C^{2}[0, b], \quad\left|h^{\prime}\right|<1 \text { in }[0, b], \quad h>0 \text { in }(0, b), \\
h(x)=\mu x \quad \text { in }\left[0, a_{0}\right] \text { für ein } a_{0} \text { mit } 0<a_{0}<b, \\
h(b)=0, \quad h^{\prime}(b) \neq 0 .
\end{gathered}
$$

Die auf $\dot{G}$ vorgegebenen Randwerte seien

(1) $u(x, 0)=g_{1}(x)$ und $u(x, h(x))=g_{2}(x) \quad$ für $0 \leqq x \leqq b$, mit

$$
g_{1}, g_{2} \in C^{2}[0, b], \quad g_{1}(0)=g_{2}(0), \quad g_{1}(b)=g_{2}(b) .
$$

Die Differentialgleichung sei

$$
u_{x x}-u_{y y}=g
$$

mit $g \in C^{0}(\bar{G})$ und $g_{x}$ existiere mit $g_{\mathfrak{r}} \in C^{0}(\bar{G})$.

Die erste Randwertaufgabe (1), (2) über $G$ [2] führt auf die Integralgleichung

$$
\int_{x-h(x)}^{x+h(x)} v(\xi) d \xi=f(x) \quad \text { für } \quad 0<x<b,
$$

mit

und

$$
\begin{gathered}
v(x):=u_{y}(x, 0), \\
f(x):=2 g_{2}(x)-e(x, h(x))
\end{gathered}
$$


$\epsilon(x, y):=g_{1}(x+y)+g_{1}(x-y)-\int_{0}^{y} \int_{\eta+x-y}^{-\eta+x+y} g(\xi, \eta) d \xi d \eta \quad$ für $(x, y) \in \bar{G}$.

Die Integralgleichung (3) hat nach [2] zum Beispiel eine Lösung

$$
v \in C^{1}(0, b] \text {. }
$$

In entsprechender Weise hat (3) eine Lösung

$$
v_{1} \in C^{1}[0, b) .
$$

Aus $v$ folgt [2]

$$
u(x, y)=\frac{1}{2} e(x, y)+\frac{1}{2} \int_{x-y}^{x+y} v(\xi) d \xi
$$

als Lösung $u \in C^{2}(\bar{G}-(0,0))$ der ersten Randwertaufgabe und entsprechend $u_{1} \in C^{2}(\bar{G}-(b, 0))$.

Satz. Mit

$$
\begin{gathered}
q:=\frac{1+\mu}{1-\mu}, \quad \lambda_{k}:=\frac{2 \pi k}{\log q}, \\
\alpha_{k}:=\frac{2}{\log q} \int_{(1-\mu) a_{0}}^{(1+\mu) a_{0}}\left(v(\xi)-v_{1}(\xi)\right) \cos \left(\lambda_{k} \log \xi\right) d \xi
\end{gathered}
$$

und entsprechender sin-Formel für $\beta_{k}(k=1,2,3, \ldots)$ und

ist

$$
G_{0}:=\left\{(x, y) \in G \mid x+y<(1+\mu) a_{0}\right\}
$$

$$
\begin{aligned}
u=u_{1}+\sum_{k=1}^{\infty}\{ & \frac{\alpha_{k}}{\lambda_{k}} \cos \left(\frac{\lambda_{k}}{2} \log \left(x^{2}-y^{2}\right)\right) \sin \left(\frac{\lambda_{k}}{2} \log \frac{x+y}{x-y}\right) \\
& \left.+\frac{\beta_{k}}{\lambda_{k}} \sin \left(\frac{\lambda_{k}}{2} \log \left(x^{2}-y^{2}\right)\right) \sin \left(\frac{\lambda_{k}}{2} \log \frac{x+y}{x-y}\right)\right\}
\end{aligned}
$$

für $(x, y) \in G_{0}$ eine explizite Darstellung des additiven singulären Anteils von $u$ in $(0,0)$.

Beweis. Nach den Eigenschaften von $v$ und $v_{1}$ ist

$$
\int_{x-h(x)}^{x+h(x)}\left(v(\xi)-v_{1}(\xi)\right) d \xi=0 \quad \text { für } 0<x<b .
$$

Für $0<x \leqq a_{0}$ und mit 


$$
\begin{gathered}
\tau:=\frac{2 \pi \log \xi}{\log q}, \quad W(\tau):=q^{\tau /(2 \pi)}\left(v\left(q^{\tau /(2 \pi)}\right)-v_{1}\left(q^{\tau /(2 \pi)}\right)\right) \\
t:=\frac{2 \pi \log (1+\mu) x}{\log q}
\end{gathered}
$$

folgt

$$
\int_{t-2 \pi}^{t} W(\tau) d \tau=0 \quad \text { für } \quad t \leqq t_{0}:=\frac{2 \pi \log (1+\mu) a_{0}}{\log q} .
$$

Also gilt für $W$ die Funktionalgleichung

$$
W(\tau)=W(\tau-2 \pi)
$$

der periodischen Funktionen für $\tau \leqq t_{0}$. Nach (4) und (5) ist auch $W \in C^{1}\left(\left[t_{0}-2 \pi, t_{0}\right]\right), \quad W\left(t_{0}-2 \pi\right)=W\left(t_{0}\right), \quad W^{\prime}\left(t_{0}-2 \pi\right)=W^{\prime}\left(t_{0}\right)$.

Daher folgt durch Fourierentwicklung und wegen (8), daß

$$
\begin{aligned}
v(\xi)-v_{1}(\xi)= & \frac{1}{\xi} \sum_{k=1}^{\infty}\left\{\alpha_{k} \cos \left(\lambda_{k} \log \xi\right)+\beta_{k} \sin \left(\lambda_{k} \log \xi\right)\right\} \\
& \text { für } 0<\xi \leqq(1+\mu) a_{0}
\end{aligned}
$$

gleichmäßig konvergiert. Nach der Bestimmung von $u$ und $u_{1}$ gemäß (6) folgt die Behauptung durch Berechnung von

$$
\frac{1}{2} \int_{x-y}^{x+y}\left(v(\xi)-v_{1}(\xi)\right) d \xi \quad \text { für } \quad(x, y) \in G_{0}
$$

unter Beachtung von $u_{1} \in C^{1}\left(\bar{G}_{0}\right)$.

Diese Arbeit sei Herrn Ilppo Simo Louhivaara aus Anlaß meiner Gastzeit in Jyväskylä in Danbarkeit gewidmet.

Technische Universität Braunschweig

Institut A für Mathematik

D-3300 Braunschweig

Deutschland 


\section{Literatur}

[1] Fichera, G.: Studio delle singolarità della soluzione di un problema di Dirichlet per l'equazione $u_{x y}=0$. - Atti Accad. naz. Lincei, Rend., Cl. Sci. fis. mat. natur., VIII. Ser. 50, 1971, S. $6-17$.

[2] Jaenicke, J.: Lösung der ersten Randwertaufgabe einer partiellen Differentialgleichung vom hyperbolischen Typus. - Math. Z. 105, 1968, S. 72-86. 